(C2006 IEEE. Personal use of this material is permitted. However, permission to reprint/republish this material for advertising or promotional purposes or for creating new collective works for resale or redistribution to servers or lists, or to reuse any copyrighted component of this work in other works must be obtained from the IEEE 


\title{
Quantifying Risk in Financial Terms According to Context and Time for Decision Making
}

\author{
Omar Khadeer Hussain ${ }^{1}$, Elizabeth Chang ${ }^{1}$ and Tharam S. Dillon ${ }^{2}$ \\ ${ }^{1}$ School of Information Systems, Curtin University of Technology, Perth, Australia \\ \{Omar.Hussain, Elizabeth.Chang\}@cbs.curtin.edu.au \\ ${ }^{2}$ Faculty of Information Technology, University of Technology, Sydney, Australia \\ tharam@it.uts.edu.au
}

\begin{abstract}
One of the characteristic of Risk is the possible loss that could be incurred in an interaction. In a peer-topeer financial interaction the loss incurred is usually the financial loss to the resources of the trusting peer that are involved in the interaction. Hence a way for the trusting peer to analyse the Risk in interacting with any trusted peer in order to decide wether to interact with it or not, is to determine the possible loss to its resources that are involved in the interaction. In this paper we will propose a methodology by which the trusting peer can determine the possible loss that could be incurred to it in interacting with a trusted peer.
\end{abstract}

\section{Introduction}

In almost every interaction the trusting peer would like to analyze the Risk that could be present in its interaction with the trusted peer for its peace of mind. By analyzing the Risk in the interaction it determines the possible loss to its resources in interacting with the trusted peer and then can take a decision of whether to interact with the trusted peer or not. The Australian and New Zealand Standard on Risk Management, AS/NZS 4360:2004 too states that Risk Identification is the heart of Risk Management [1]. Hence Risk should be identified in order to analyze and manage it better. Risk analysis is the science of evaluating Risks resulting from past, current, anticipated or future activities. The use of these evaluations includes providing information for determining regulatory actions to limit Risk and for decision making. Risk analysis is an interdisciplinary science that relies on epidemiology and laboratory studies, collection and exposure of field data and computer modeling. Risk can be seen as a combination of:

a) The uncertainty of the outcome and

b) The cost of the outcomes when it occurs, usually the loss incurred, which is related to Risk.
In the literature different definitions have been defined for Risk. All those definitions highlight and emphasize on the possible loss in an interaction. To mention some of such definitions March et al define Risk more by the magnitude of the value of the outcome rather than by taking its likelihood [2]. Luhmann defines Risk in a transaction where the possible damage might be more than the advantage sought [3]. Mayer et al conclude that Risk is present in the transaction only if the negative outcome outweighs the positive outcome at the end of the transaction [4]. In contrast to this definition, Rousseau et al measures Risk as the potential negative consequence and probability of failure [5]. Sztompka defines Risk as the probability of the loss of the resources invested [6]. Grazioli et al views Risk as the consumers' perception of the uncertainty and adverse consequences of engaging in an activity [7]. Cheung et al define Risk as having two dimensions; one related to the uncertainty or probability of loss notion and the other related to a consequence of the importance of the notion of loss [8]. We define Risk in the context of peer-to-peer interaction as the likelihood that the trusted peer might not act as expected according to the trusting peer's expectations in a given context and at a particular time once the interaction begins, resulting in the loss of $\$$ and the resources involved in the interaction [9]. The possible loss in the interaction might be related to the resources which the trusting peer has at stake in its interaction with the trusted peer. In a peer-to-peer financial interaction the trusting peer's financial resources are at stake in the interaction.

Risk is important in the study of behavior in ecommerce, because there is a whole body of literature based in rational economics that argues that the decision to buy is based on the Risk-adjusted costbenefit analysis [13]. Thus it commands a central role in any discussion of e-commerce that is related to a transaction. The need to distinguish between the likelihood and magnitude of Risk is important. This can be explained by taking the empirical evidence in a 
web based sale. For example the likelihood of selling an item on the web decreases as the cost of the product increases. For higher cost items, the web does not tend to act as a medium to buy, but as a means for providing information and vice versa for lower cost items. The likelihood of a negative outcome might be the same in both transactions, but the magnitude of loss will be greater in a higher cost transaction. Therefore, the relative reluctance of the customers to buy high cost items on the Internet, compared to the demand for lower cost items, would be consistent with the idea that magnitude of potential loss defines perception of Risk, and not likelihood of loss [2]. Risk plays a central role in deciding whether to proceed with a transaction or not. It can broadly be defined as an attribute of decision making that reflects the variance of its possible outcomes. Peer-to-Peer communications are being described as the next generation of the Internet [14]. Some researchers are proposing architectures for integrating web services with Peer-to-Peer communication agents like Gnutella [15-18].However, Peer-to-Peer communications suffer from some disadvantages and Risk in the transaction is one of them. Hence we need to develop a mechanism by which we can over come this disadvantage so that they can be used effectively with what ever service they is being integrated with.

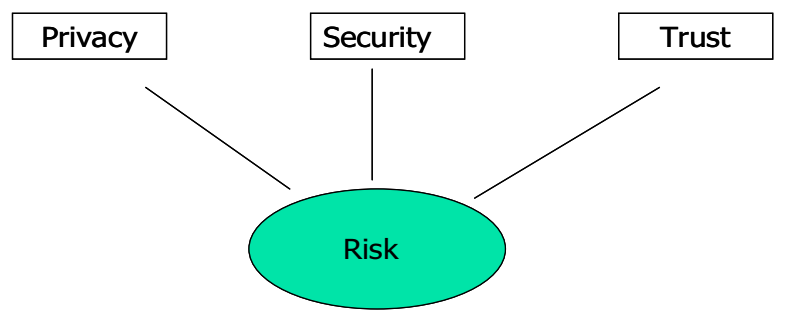

Figure 1. The association of Risk with Privacy, Security and Trust

The inclusion of Risk in the study of behavior in ecommerce transaction is important because there is a large volume of literature based in rational economics that argues that the decision to proceed with the transaction is based on the Risk adjusted cost benefit analysis and analyzing Risk in the transaction is really important with the widespread use of the Internet, particularly with the advent of business and ecommerce transactions and the integration of Peer-toPeer communications with web services [19].

Hence one of the main decision making factor for the trusting peer to decide whether to interact with a trusted peer or not is by determining the possible loss in its resources in interacting with it. By the loss in its resources we mean the financial loss that could be incurred to the trusting peer in interacting with a trusted peer. In this paper we propose such a methodology by which the trusting peer can determine beforehand the loss to its resources in interacting with a trusted peer.

\section{Related Work}

In order to analyze the Risk that could be present in an interaction we defined the term Riskiness in Hussain et al [9]. Riskiness is defined as the numerical value that is assigned to the trusted peer on the Riskiness scale by the trusting peer after its interaction. The Riskiness scale as shown in figure 2 depicts different levels of Risk that could be possible in an interaction. The numerical value assigned to the trusted peer on the Riskiness scale shows the level of Risk that was present in their interaction.

The Riskiness value is assigned to the trusted peer by the trusting peer after assessing the level of uncommitment in the trusted peer's actual behaviour with respect to the expected behaviour. The methodology by which the trusting peer determinates the un-committed behaviour in an interaction by the trusted peer and assigns it with a Riskiness value that is deserves is defined in Hussain et al [9].

Hence any future trusting peer can decide wether to interact or not with a particular trusted peer by analysing the trusted peer's Riskiness value in the particular context of its interaction. If the future trusting peer analysing the Riskiness value of the trusted peer has any previous interaction history with the trusted peer in the same time space and in the same context as its future interaction, then it can make a

\begin{tabular}{|c|c|c|c|}
\hline $\begin{array}{c}\text { Riskiness } \\
\text { Levels }\end{array}$ & \begin{tabular}{|c}
$\begin{array}{c}\text { Magnitude of } \\
\text { Risk }\end{array}$ \\
\end{tabular} & $\begin{array}{l}\text { Riskiness } \\
\text { Value }\end{array}$ & Star Rating \\
\hline Unknown Risk & - & -1 & Not Displayed \\
\hline Totally Risky & $\begin{array}{c}91-100 \% \text { of } \\
\text { Risk }\end{array}$ & 0 & Not Displayed \\
\hline $\begin{array}{c}\text { Extremely } \\
\text { Risky }\end{array}$ & $\begin{array}{l}71-90 \% \text { of } \\
\text { Risk }\end{array}$ & 1 & \\
\hline Largely Risky & $\begin{array}{l}51-70 \% \text { of } \\
\text { Risk }\end{array}$ & 2 & \\
\hline Risky & $\begin{array}{l}26-50 \% \text { of } \\
\text { Risk }\end{array}$ & 3 & \\
\hline $\begin{array}{l}\text { Largely } \\
\text { UnRisky }\end{array}$ & $\begin{array}{c}11-25 \% \text { of } \\
\text { Risk }\end{array}$ & 4 & \\
\hline UnRisky & $\begin{array}{c}0-10 \% \text { of } \\
\text { Risk }\end{array}$ & 5 & \\
\hline
\end{tabular}

Figure 2. Showing the Riskiness scale and its associated levels

decision wether to interact or not with the trusted peer based on its previous interaction history with the trusted peer. If on the other hand the future trusting peer does not have any previous interaction history with the trusted peer, then it can determine its 
Riskiness value by soliciting for recommendations from other peers in the context of its interaction. The peers replying back with the recommendations are called as the Recommending Peers. As mentioned in Hussain et al [10] the recommending peers communicate their recommendations in the form of a Risk Set. The Risk set is an ordered way of communicating recommendations so that it is easier for the trusting peer to interpret and analyse them. The format of the Risk set is defined in Hussain et al [10]. Once the future trusting peer gets the recommendations, then it can assimilate them and determine the Riskiness value of the trusted peer. Based on the Riskiness value achieved it can decide wether to interact or not with the trusted peer.

But as mentioned earlier the Riskiness value to a trusted peer is assigned by the trusting peer after assessing the level of un-commitment in its actual behaviour with respect to the expected behaviour. Expected behaviour is defined by the trusting peer before its interaction with the trusted peer, according to the criteria of its interaction. Hence the un-committed behaviour in the interaction by the trusted peer is determined according to the criteria of the trusting peer's or recommending peer's (while giving recommendation) interaction and subsequently the Riskiness value that is assigned to the trusted peer too is according to its criteria in the interaction. It is highly possible that any other possible trusting peer who wants to interact with a trusted peer and is analysing its Riskiness values might have different criteria in its interaction even thought it is in the same context. Hence Riskiness values like these will not be of any use to the future trusting peer analysing the Riskiness of the trusted peer as they don't match with its criteria. Similarly the Riskiness value that the recommending peers recommend might be according to the criteria of their interaction. It is highly possible that the criteria of a recommending peer communicating a recommendation for a trusted peer and a future trusting peer soliciting for recommendation for the same trusted peer might not be the same, even though they are in the same context. Hence it would be baseless for the future trusting peer to assimilate and determine the Riskiness value of the trusted peer from recommendations whose criteria are not similar to those in its interaction.

In order to overcome this disadvantage we defined a Risk based Decision Support System (RDSS) in Hussain et al [11]. This system classifies the recommendations according to its trustworthiness and then assimilates the trustworthy and unknown recommendations according to their time and the criteria of the trusting peer in its future interaction with the trusted peer. Hence the Riskiness value that the future trusting peer gets for a possible trusted peer is according to the criteria of its interaction with it. Further it omits the un-trustworthy recommendations and reduces the Risk of assimilating the data which is not trustworthy. If the future trusting peer has to decide among a set of possible trusted peers, with which peer to interact with, then it can use the RDSS to determine the Riskiness value of each possible trusted peer according to the criteria of its interaction and then decide on one among them. We will discuss briefly the RDSS system in the next section.

\section{Risk based Decision Support System}

As mentioned in section 2, the trusting peer might have to decide from a set of possible trusted peers with whom to interact with in an interaction. Further if the trusting peer has not interacted with any of the trusted peers before then it doesn't know the level of Risk that could be present in the interaction and hence it would be difficult for it to decide on with which trusted peer to interact with. A solution to this would be for the trusting peer to assess the Risk involved in dealing with the trusted peers according to the context, time and criteria of the trusting peer's interaction. Depending on analysis the trusting peer can easily decide with which trusting peer to interact with.

The Risk based decision support system while assimilating the recommendations also considers the following:

1. The time spot at which the recommending peer interacted with the trusted peer. As mentioned in the previous section, Risk is dynamic and hence the trusting peer should give more weight to recommendations which are in the same time slot.

2. The recommendations from peers who are either trustworthy or unknown recommenders and discard the recommendations from those peers who give un-trustworthy recommendations. As discussed in Hussain et al [20], the peer whose Riskiness value while giving recommendations is with in the range of $(-1,1)$ is said to be giving trustworthy recommendations.

The Riskiness value of the trusted peer in each criterion of the recommending peer's interaction can be determined by assimilating the recommendations by using equation 1. The final Riskiness value of the trusted peer, in the trusting peer's criterion can be achieved by weighting the trusted peer's Riskiness value of each criterion with respect to its significance according to the trusting peer.

The Riskiness value of a particular trusted peer ' $\mathrm{P}$ ' in each criterion $\mathrm{C}\left(\mathrm{R}_{\mathrm{PC}}\right)$ can be determined after assimilating the recommendations by using the following formulae: 
Riskiness value of the trusted peer ' $\mathrm{P}$ ' in Criterion $\mathrm{C}\left(\mathrm{R}_{\mathrm{PC}}\right)=$

$$
\begin{aligned}
& \left(\alpha * \left(\left(\frac{1}{N} * \mid \gamma *\left(\sum_{i=1}^{N} \mathrm{RRP}_{\mathrm{i}} * \text { Commitment Level }_{\mathrm{c}}\right) \mid\right)+\right.\right. \\
& \left.\left.\left(\frac{1}{K} * \mid \delta *\left(\sum_{l=1}^{K} \mathrm{RRP}_{1} * \text { Commitment Level }_{\mathrm{c}}\right) \mid\right)\right)\right) \\
& + \\
& \left(\beta * \left(\left(\frac{1}{J} * \gamma\left(\sum_{o=1}^{J} \text { Commitment Level }_{\mathrm{c}}\right)\right)+\right.\right. \\
& \left.\left.\left(\frac{1}{M} * \delta\left(\sum_{q=1}^{M} \text { Commitment Level } \mathrm{c}_{\mathrm{c}}\right)\right)\right)\right)
\end{aligned}
$$

Equation--------1

where $R P_{i}$ is the Riskiness value of the trustworthy recommending peer $i$ whose recommendation is in the same time slot of the trusting peer's interaction,

$\mathrm{RRP}_{1}$ is the Riskiness value of the trustworthy recommending peer 1 , whose recommendation is not in the same time slot of the trusting peer's interaction,

Commitment level ${ }_{c}$ is the level of commitment by the trusted peer in the particular criterion ' $c$ ' as recommended by the recommending peer in its recommendations,

$\mathrm{N}$ and $\mathrm{K}$ are the number of trustworthy recommendations classified according to the same and different time slots respectively,

$\mathrm{J}$ and $\mathrm{M}$ are the number of unknown recommendations classified according to the same and different time slots respectively,

$\gamma$ and $\delta$ are the weights attached to the parts of the equation which give more weight to recommendations which are in the same time slot as compared to the ones which are not. $\gamma>\delta$ and $\gamma+\delta=1$,

$\alpha$ and $\beta$ are the weights attached to the parts of the equation which will give more weight to the recommendation from the trustworthy recommending peers as compared to the unknown recommending peers. $\alpha>\beta$, and $\alpha+\beta=1$.

The first part of the above equation calculates the Riskiness value of the trusted peer ' $p$ ' in a criterion 'c ' by taking the recommendations of the trustworthy recommending peers and the second part calculates the Riskiness value of the same trusted peer in the same criterion by taking the recommendations of the unknown recommending peers. The recommendations from the untrustworthy recommending peers are left out and not considered. Further the Riskiness value determination of the trusted peer by taking the recommendations of the trustworthy and the unknown recommending peers too is done in two parts according to the time slot of the recommendations. The trusting peer gives more weight to the recommendations which are in the same time slot of its interaction as compared to the one which are in a different time slot. Those weights are represented by $\gamma$ and $\delta$ respectively. In order to give more importance to the recommendations from the trustworthy recommending peers as compared to the recommendations from the unknown recommending peers, weights are attached to the two parts of the equation. These weights are represented by $\alpha$ and $\beta$ respectively. It depends upon the trusting peer on how much weight does it want to give to each recommendation. By multiplying the Riskiness value of the recommending peer (RRP) with the commitment level that it is suggesting for a criterion we are getting the accurate recommendation according to its Riskiness.

As mentioned earlier any recommending peer whose Riskiness value while giving recommendations is with in the range of $(-1,1)$ is said to be a trustworthy recommending peer. So it is possible that the Riskiness value for the trusted peer in a criterion ' $c$ ' calculated from the trustworthy recommendations might come negative. We take the range of $(-1,1)$ to determine whether the recommendation is trustworthy or not and once it has been determined, it should not have any effect in determining the final Riskiness value of the trusted peer in a criterion by assimilating the recommendations. Hence we apply the mod operator in equation 1 to the first part of the equation which determines the Riskiness of the trusted peer in a criterion ' $c$ ' by taking the trustworthy recommendations.

In order to map the Riskiness value $\left(\mathrm{R}_{\mathrm{PC}}\right)$ of the trusted peer ' $p$ ' in a criterion ' $c$ ' on the riskiness scale (RS), it should be multiplied by 5 . Hence Riskiness value of the trusted peer ' $p$ ' in a criterion ' $c$ ', mapped to the Riskiness scale $\left(\mathrm{R}_{\mathrm{PRSC}}\right)$ is:

$$
\mathrm{R}_{\mathrm{PRSC}}=\mathrm{ROUND}\left(\mathrm{R}_{\mathrm{PC}} * 5\right) \quad \text { Equation--------2 }
$$

When the Riskiness value in each criterion of the trusting peer's interaction has been determined on the Riskiness scale for the trusted peer by assimilating the recommendations, then the final Riskiness value of the trusted peer in the interaction can be determined by weighing the individual Riskiness value of each criterion according to its significance, depending on the trusting peer. The levels of significance for each criterion (Sc) are shown in table 1. All the criteria of an interaction will not be of equal importance or significance. Some criteria might play an important role in the completion of the interaction and some might not be as crucial as others. The significance of 
each criterion in an interaction might depend on the degree to which it influences the successful outcome of the interaction according to the trusting peer.

Hence the final Riskiness value $(\mathrm{CR} p)$ of the trusted peer ' $p$ ' as determined by the trusting peer ' $A$ ' according to its criteria and significance of each criterion in the interaction by asking recommendations from other peers can be calculated as:

\begin{tabular}{|c|l|}
\hline $\begin{array}{l}\text { Significance level of the } \\
\text { Criterion (Sc) }\end{array}$ & $\begin{array}{l}\text { Significance Rating and } \\
\text { Semantics of the level }\end{array}$ \\
\hline 1 & Minorly Significant \\
\hline 2 & Moderately Significant \\
\hline 3 & Largely Significant \\
\hline 4 & Majorly Significant \\
\hline 5 & $\begin{array}{l}\text { Highly or Extremely } \\
\text { Signifcant }\end{array}$ \\
\hline
\end{tabular}

Table 1 showing the significance level of each criterion

$$
\mathrm{CR}_{\mathrm{p}}=\operatorname{ROUND}\left(\frac{1}{\sum_{c=1}^{n} S c}\left(\sum_{c=1}^{n} \mathrm{Sc} * \mathrm{R}_{\mathrm{PRSC}}\right)\right)
$$

$$
\text { Equation-------3 }
$$

Where Sc represents the significance of the criterion 'c'

$\mathrm{R}_{\mathrm{PRSC}}$ represents the Riskiness value of the trusted peer ' $p$ ' in criterion ' $c$ ' on the Riskiness scale

$\mathrm{n}$ is the number of criterions in the interaction.

It should be noted that the Riskiness value of the trusted peer $\left(\mathrm{CR}_{\mathrm{p}}\right)$ determined by assimilating the recommendations should be set to 0 if it is less than 0 , as the Riskiness scale ranges from 0 to 5 with a value of -1 as Unknown Risk .

Finally when the trusting peer ' $A$ ' calculates the Riskiness values of the trusted peers ' $B$ ' and ' $C$ ' according to the criterions of its interaction by using the above proposed concept, then it can easily decide with which trusted peer to interact with depending on their Riskiness values.

But it is possible that the Riskiness value of each possible trusted peer that is determined by assimilating the recommendations according to the criteria of the future trusting peer by RDSS might be the same for one or more possible trusted peers. Then the factor for the future trusting peer to decide with which trusted peer to interact with, shifts to another characteristic of Risk, namely the possible loss that could be incurred in the interaction when interacting with a particular peer. In a peer-to-peer financial interaction the possible loss is the possible financial loss to the trusting peer in interacting with the trusted peer. The trusting peer can decide with which peer to interact with by determining the possible loss to its resources in interacting with each possible trusted peer.

The financial loss to a trusting peer in an interaction is dependent on:

1. Its previous interaction history with the trusted peer if any, or on the reputation of the trusted peer determined by soliciting for recommendations from other peers according to the criteria of its interaction, 2. Predicting the future Riskiness value of the trusted peer till the time space of the trusting peer's interaction according to its previous Riskiness value,

3. Willingness of the trusting peer in interacting with the trusted peer,

4. Familiarity of the trusting peer with the medium of interaction,

5. Familiarity of the trusting peer with the trusted peer,

6. Nature of the trusting peer.

All these factors have to be considered while determining the possible financial loss in the trusting peer's resources in interacting with the trusted peer. In the next section we will explain each of these factors in detail and define the metrics which are used to quantify each of these factors and express them numerically.

\section{Factors for Determining Financial Loss in an Interaction}

\subsection{Previous Interactions or Considering Reputation from other peers}

The outcome of previous interactions, if any, between the trusting peer and the trusted peer will help in analyzing the Risk that could be present in the future interactions between them. Depending on the outcome of the previous interaction, the trusting peer might be able to make a decision to trust the trusted peer or not in its future interaction. If the outcome of the previous interaction was positive and it concluded according to the expected behavior, then the trusting peer might have some belief in the trusted peer and may proceed with any future interaction with it. Consequently the Risk associated with this interaction might not be high, as there is some belief present among them. On the contrary, if the outcome of the previous interaction was negative then the trusting peer may have some doubts about proceeding with any future interaction with the trusted peer, and fears the Risk involved in the interaction might be high.

If there is no previous interaction history between the trusting peer and the trusted peer, then the trusting peer can analyse the Risk that could be present in its interaction with the trusted peer by inquiring for its reputation in the particular context its interaction. As 
discussed earlier reputation of a trusted peer can be determined by soliciting for its recommendations from other peers and later assimilating them.

We represent the Riskiness value of the trusted peer before starting an interaction, that is achieved either by the previous interaction history of the trusting peer with the trusted peer or by soliciting for its recommendations from other peers by the metric Pre Trusted Peer. The value of the metric Pre Trusted Peer is between $(0,5)$ on the Riskiness scale.

\subsection{Predicting the future Riskiness value of the trusted peer}

Risk varies according to time. It is not possible for a trusting peer to have the same impression of the trusted peer that it had at a particular time throughout its interaction. Hence the trusting peer in order to determine the possible financial loss in an interaction accurately should take into consideration the future Riskiness value of the trusted peer till the time slot of their interaction. The future Riskiness value for the trusted peer is predicted based on the recommendations achieved from the recommending peers according to the criteria of the trusting peer's interaction. The process of classifying the time of the trusting peer's interaction with the trusted peer into different time slots and predicting the future Riskiness value of the trusted peer in each time slot is defined in Hussain et al [12].

We represent the future Riskiness value of the trusted peer within the time space of the trusting peer's interaction by the metric Fut Trusted Peer. The predicted future Riskiness value of the trusted peer Fut Trusted Peer will be in the range of $(0,5)$ on the Riskiness scale.

\subsection{Willingness of the Trusting peer in interacting with the trusted peer}

The financial loss in an interaction also depends on the contentment / willingness of the trusting peer in dealing with the trusted peer. It depends on the degree of how much comfortable is the trusting peer in dealing with the trusted peer. The trusting peer will be more comfortable in dealing with the trusted peer if it has a favourable previous interaction history with the trusted peer and there was a high degree of parallelism between the expected and actual behaviours. The degree of parallelism between Expected and Actual behaviour helps in finding the belief that the trusting peer has for the trusted peer. The degree of parallelism between the expected behaviour and the actual behaviour is represented by the Riskiness value that is assigned after the interaction. The greater degree of parallelism between these two behaviours indicates that the trusted peer has acted according to the expected behaviour and the Risk involved in the interaction was less. Hence the trusting peer might be contented in interacting with the trusted peer and might proceed with another interaction with it in the near future, as they have established a significant level of trust between them.

We define the willingness of the trusting peer to interact with the trusted peer by the metric Will Interaction. In order to measure the willingness of the trusting peer in interacting with the trusted peer we define 3 levels of the metric Will Trusting Peer. Those levels are defined in table 2 .

\subsection{Familiarity of the trusting peer with the medium of interaction}

The possible degree of financial loss in the trusting peer's resources also varies according to the familiarity of the trusting peer towards the medium of interaction with the trusted peer. If the trusting peer is familiar with the medium in which it is going to interact with the trusted peer then it will ease the Risk that could be present in the interaction.

We represent the familiarity of the trusting peer with the medium of interaction by the metric Fam Medium. In order to measure the familiarity of the trusting peer with the medium of interaction we define two levels for the metric Fam Medium. Those levels are defined in table 3 .

\subsection{Familiarity of the trusting peer with the trusted peer}

If the trusting peer and the trusted peer are familiar with each other as a result of past interactions between them, then the Risk that could be present in their future interactions can be analyzed easily depending on the Riskiness values that were assigned in their previous interactions. Hence in order to determine the amount of financial loss in an interaction between the trusting peer and the trusted peer it is important to consider if the trusting peer is familiar with the trusted peer or not. The familiarity of the trusting peer with the trusted peer is represented by the metric Fam Trusted Peer. In order to measure the familiarity of the trusting peer with the trusted peer we define two levels of the metric Fam ${ }_{\text {Trusted Peer }}$ in table 4.

\subsection{Nature of the trusting peer}

One of the factors which vary Risk in an interaction is the nature and thinking attitude of the trusting peer, which decides on how it will act and react in certain 
situations. The nature of the trusting peer plays an important role to decide whether to proceed with the interaction or not, thus helping in determining the financial loss that could be involved in an interaction.

If the trusting peer has an impatient nature of acting in haste, then there is a possibility that he might proceed with the interaction without looking at the previous history of the trusted peer or soliciting for recommendations, hoping to achieve the desired outcome as soon as possible. The Risk associated in these interactions might be high.

It might be possible that the trusting peer might care for personal values and start an interaction. For example let us suppose that the trusting peer and the trusted peer might have dealt before successfully in a different context and in a different interaction. Now they are dealing again in a context and in an interaction in which the trusted peer does not have any experience before. Yet the trusting peer might care for personal values and might be ready to take the extra Risk involved and proceed with the interaction.

On the contrary the trusting peer might have a cautious nature of proceeding according to the results and feedbacks of the previous interactions of the trusted peer. Then in this case it might not be willing to trust the trusted peer in any other context in which it does not have any experience before. He might feel the Risk involved in such interaction could be high and might not proceed ahead in interacting with him. Hence the Risk too decreases according to the trusting peer's decision. If the trusting peer decides to go ahead in an interaction according to the correct way, i.e. in a logical fashion and not care about the personal values then it will be making the decision to proceed with the interaction only if the expected advantages outweigh the negative factors. The Risk involved in such interactions might be less as compared to the other ones.

Hence the trusting peer might decide to proceed in the interaction or not, depending on its nature and hence it is important to take that into consideration while determining the financial loss in an interaction. The nature of the trusting peer is represented by the metric Nat Trusting Peer. We define 3 levels of the metric Nat Trusting Peer in order to determine the nature of the trusting peer. Those levels are defined in table 5 .

\begin{tabular}{|c|l|}
\hline $\begin{array}{l}\text { Level of the } \\
\text { Metric Will Trusting } \\
\text { Peer }\end{array}$ & \multicolumn{1}{c|}{ Semantics of the level } \\
\hline 0 & $\begin{array}{l}\text { The trusting peer is not at all willing to deal } \\
\text { with the trusted peer }\end{array}$ \\
\hline 1 & $\begin{array}{l}\text { The trusting peer is comfortable in dealing } \\
\text { with the trusted peer but would prefer in } \\
\text { dealing with other peers if possible }\end{array}$ \\
\hline 2 & $\begin{array}{l}\text { The trusting peer is totally comfortable in } \\
\text { dealing with the trusted peer }\end{array}$ \\
\hline
\end{tabular}

Table 2 showing the levels for the metric Will ${ }_{\text {Interaction }}$

\begin{tabular}{|c|l|}
\hline $\begin{array}{c}\text { Level of the } \\
\text { Metric Fam }\end{array}$ & \multicolumn{1}{c|}{ Semantics of the level } \\
\hline 0 & $\begin{array}{l}\text { The trusting peer is not familiar with the } \\
\text { medium of the interaction }\end{array}$ \\
\hline 1 & $\begin{array}{l}\text { The trusting peer has previous experience } \\
\text { and is familiar with the medium of the } \\
\text { interaction }\end{array}$ \\
\hline
\end{tabular}

Table 3 showing the level for the metric Fam Medium

\begin{tabular}{|l|l|}
\hline $\begin{array}{l}\text { Level of the } \\
\text { metric Fam Trusted } \\
\text { Peer }\end{array}$ & \multicolumn{1}{c|}{ Semantics of the level } \\
\hline 0 & $\begin{array}{l}\text { The trusting peer has not interacted with the } \\
\text { trusted peer before and is not at all familiar } \\
\text { with it }\end{array}$ \\
\hline 1 & $\begin{array}{l}\text { The trusting peer has previous interaction } \\
\text { history with the trusted peer and is familiar } \\
\text { with it. }\end{array}$ \\
\hline
\end{tabular}

Table 4 showing the level for the metric Fam Trusted Peer

\begin{tabular}{|c|l|}
\hline $\begin{array}{c}\text { Level of the } \\
\text { metric Nat Trusting } \\
\text { Peer }\end{array}$ & \multicolumn{1}{c|}{ Semantics of the Level } \\
\hline 0 & $\begin{array}{l}\text { The trusting peer is impulsive in nature and } \\
\text { acts in haste. It prefers proceeding in an } \\
\text { interaction even if there is high Risk or } \\
\text { without analyzing the Risk that could be } \\
\text { present in an interaction. }\end{array}$ \\
\hline 1 & $\begin{array}{l}\text { The trusting peer is conservative in nature } \\
\text { and prefers taking some Risk. It might also } \\
\text { care for personal values. }\end{array}$ \\
\hline 2 & $\begin{array}{l}\text { The trusting peer is determined in nature and } \\
\text { will proceed in the interaction only if the } \\
\text { expected advantages outweigh the negative } \\
\text { factors. }\end{array}$ \\
\hline
\end{tabular}

Table 5 showing the level for the metric Nat Trusting Peer

\section{Determining the possible Financial Loss in an Interaction}

In order to determine the possible financial loss in an interaction, the trusting peer by making use of the above mentioned metrics should derive a numerical value which quantifies its possible interaction with the trusted peer. The numerical value that is derived by using the above metrics will take into consideration factors namely 
- the previous interaction of the trusting peer with the trusted peer if any,

- the reputation of the trusted peer determined by assimilating the recommendations,

- the predicted future Riskiness value of the trusted peer within the time slot of its interaction with the trusting peer,

- the willingness of the trusting peer in dealing with the trusted peer,

- familiarity of the trusting peer with the medium of interaction,

- familiarity of the trusting peer with the trusted peer,

- nature of the trusting peer which is a critical factor in deciding whether to interact or not with the trusted peer.

Hence the numerical value quantifying the possible interaction of the trusting peer with the trusted peer can be determined by adding the individual value of each metric. We represent the numerical value quantifying the possible interaction of the trusting peer with the trusted peer as Poss Interaction.

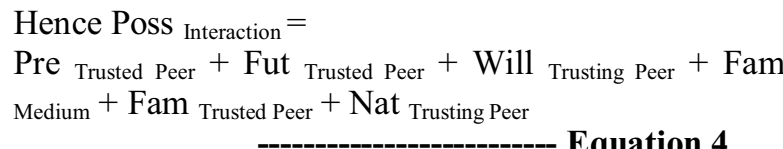
Equation 4

Once the numerical value quantifying the possible interaction between the trusting peer and the trusted peer has been determined, it should be compared with the numerical value which quantifies an interaction between the same peers with no financial loss at all to the trusting peer's resources. This value is achieved by the above defined metrics by substituting the individual values for each metric in equation 4 that would represent a totally non-risky interaction. We represent the numerical value which quantifies an interaction with no financial loss at all to the trusting peer's resources as Noloss Interaction.

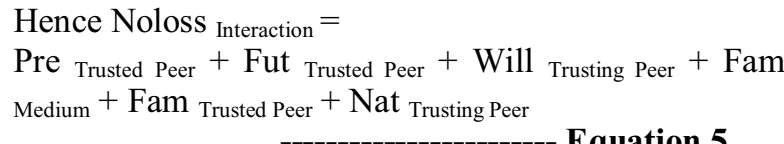

We define Loss Interaction as the metric which expresses in numerical value the possible level of loss that could be possible in an interaction. This is achieved by comparing the difference of the numerical values representing a totally non-risky interaction and the possible way in which the interaction might proceed with respect to the numerical value that expresses an interaction with no financial loss at all.
The metric Loss Interaction depicts the level of financial loss that could be possible in an interaction to the trusting peer with the trusted peer.

Hence Loss Interaction $_{\text {is determined by: }}$

Loss $_{\text {Interaction }}=\frac{\text { NolosSInteraction }- \text { PosSInteraction }}{\text { NoloSSInteraction }}$

Consequently,

Financial Loss in an interaction $=$

(Cost of the Interaction * Loss $\left.{ }_{\text {Interaction }}\right)$

Equation 7

The possible percent of loss in an interaction can be determined as:

Possible Percent of Loss Interaction $=\left(\right.$ Loss $\left._{\text {Interaction }} * 100\right)$

Equation 8

A better understanding of the concept of determining the possible financial loss to the trusting peer in interacting with a trusted peer can be achieved in the next section when we explain it by an example.

\section{Example of determining the possible Financial Loss in an Interaction}

In order to get a better understanding of the proposed model let us consider an example of determining the possible financial loss in an interaction to the trusting peer in interacting with a trusted peer by using the defined metrics.

Let us consider that a trusting peer ' $A$ ' has to interact with a trusted peer in the context of 'transporting its goods'. The trusting peer has to decide among a set of trusted peers with which particular peer to interact with. The set of possible trusted peers are Peer ' $B$ ' and Peer ' $C$ '. Let us further assume that

- The criteria in the trusting peer's interaction are $\mathrm{C} 1, \mathrm{C} 2$ and $\mathrm{C} 3$.

- The goods are of worth 15,000 \$

- The trusting peer wants to interact with the trusted peer in the period of $01 / 02 / 2006$ to $05 / 02 / 2006$.

- The trusting peer ' $A$ ' had interacted favourable with the trusted peer ' $\mathrm{B}$ ' previously in the same context but at a different time slot.

In order for the trusting peer to decide with which peer to interact with, it will divide the time space into different time slots and determine the Riskiness value of each possible trusted peer in the time slots according to its criteria by utilizing the Risk based Decision Support System (RDSS) till the time spot of its interaction. The process of dividing the time into different time slots and determining the Riskiness 
values of the trusted peers according to the criteria of the trusting peer's interaction by considering its previous interactions with the trusting peer or by assimilating recommendations from the recommending peers is mentioned in Hussain et al [11]. That Riskiness value is represented by the metric Pre ${ }_{\text {Trusted }}$ Peer.

For explanation sake let us consider that:

- The value of the metric Pre Trusted Peer for the possible trusted peers ' $\mathrm{B}$ ' and ' $\mathrm{C}$ ' is 4 and 4 respectively.

Based on Riskiness values achieved in each previous time slot, the future Riskiness value within the time space of its interaction can be predicted by using the methodology mentioned in Hussain et al [12]. The future Riskiness value is represented by Fut Trusted Peer.

Again for explanation sake let us consider that:

- The future Riskiness values predicted from the previous time slots Riskiness values for the trusted peers ' $\mathrm{B}$ ' and ' $\mathrm{C}$ ' is 4 and 4 respectively.

Hence as the past and the future Riskiness value of both the trusted peers for the time space of the trusting peer's interaction is the same, it will be difficult for the trusting peer to decide with which particular trusted peer to interact with. It can ease its process of deciding with which peer to interact, by considering the degree of possible financial loss to it in interacting with each of them.

\subsection{Determining the Financial loss to the trusting peer in interacting with possible trusted peer ' $B$ '}

Utilizing the above defined metrics to determine the financial loss to the trusting peer in interacting with the trusted peer ' $\mathrm{B}$ ':

$$
\begin{aligned}
& \text { Pre }{ }_{\text {Trusted Peer 'B' }}=4 \\
& \text { Fut Trusted Peer 'B' }=4
\end{aligned}
$$

Since the trusting peer ' $A$ ' had interacted favorably with the trusted peer ' $B$ ' it assigns a value of 2 to the metric Will ${ }_{\text {Trusting Peer }}$

As the trusting peer ' $A$ ' has previous interaction history with the trusted peer ' $\mathrm{B}$ ' it is familiar with the medium of interaction. Hence the value of Fam Medium is 1

The value of Fam Trusted Peer is 1

The trusting peer is determined in nature and the value for the metric Nat Trusting Peer is 2 .

Quantifying the possible interaction of the trusting peer with the trusted peer according to equation 4 we get:

Poss ${\text { Interaction }{ }^{\mathrm{B}} \text { ' }}=14$
Quantifying the value of the metric Noloss Interaction by using equation 5 that would represent a non-Risky interaction and hence no loss in financial terms to the trusting peer we get:

$$
\begin{aligned}
& \text { Pre }_{\text {Trusted Peer ' } \mathrm{B} \text { ' }}=5 \\
& \text { Fut }_{\text {Trusted Peer }} \text { ' } \text { ' }=5 \\
& \text { Will }_{\text {Trusting Peer }}=2 \\
& \text { Fam }_{\text {Medium }}=1 \\
& \text { Fam }_{\text {Trusted Peer }}=1 \\
& \text { Nat }_{\text {Trusting Peer }}=2 \\
& \text { Hence Noloss }_{\text {Interaction 'B' }}=5+5+2+1+1+2 \\
& \quad \text { Noloss } \\
& \text { Interaction 'B' }
\end{aligned}
$$

Determining the numerical value expressing the possible degree of loss that could be possible in an interaction by utilizing equation 6 we get:

Loss $_{\text {Interaction 'B }}=\frac{16-14}{16}$

Loss $_{\text {Interaction }}{ }^{\mathrm{B}} \mathrm{B}=0.125$

Consequently determining the Financial Loss in an interaction according to equation 7

$$
\begin{aligned}
\text { Financial Loss in interacting with peer ' } \mathbf{B} \text { ' } \\
=(15000 * 0.125) \\
=\$ \mathbf{1 8 7 5}
\end{aligned}
$$

Possible Percent of Loss ${ }_{\text {Interaction }}{ }^{\mathrm{B}} \mathrm{B}$ ' $=\left(\right.$ LosS $_{\text {Interaction }} \mathrm{C}_{\mathrm{B}}$, * 100)

Possible Percent of Loss ${\text { Interaction }{ }^{\mathrm{B}} \mathrm{B}}=12.5 \%$

\subsection{Determining the Financial loss to the trusting peer in interacting with possible trusted peer ' $C$ '}

Similarly determining the possible financial loss to the trusting peer ' $A$ ' by utilizing the metrics in interacting with the trusted peer ' $\mathrm{C}$ ' we get:

$$
\begin{aligned}
& \text { Pre }{ }_{\text {Trusted Peer }}{ }^{C} \text { ' }=4 \\
& \text { Fut }{ }_{\text {Trusted Peer }{ }^{~} \mathrm{C} \text { ' }}=4
\end{aligned}
$$

Although the trusting peer ' $A$ ' has not interacted previously with the trusted peer ' $\mathrm{C}$ ', it is comfortable in dealing with it by analyzing its past and future Riskiness values. Hence it assigns a value of 2 to the metric Will Trusting Peer.

The trusting peer ' $A$ ' has previous interaction history in this type of medium and hence the value of Fam Medium is 1

As the trusting peer ' $\mathrm{A}$ ' does not have any previous interaction history with the trusting peer ' $\mathrm{C}$ ' the value of Fam Trusted Peer is 0 
The trusting peer is determined in nature and the value for the metric Nat Trusting Peer is 2 .

Quantifying the possible interaction of the trusting peer with the trusted peer by using equation 4 we get:

Poss ${ }_{\text {Interaction }}{ }^{C} \mathrm{C}$ ' $=13$

Quantifying the value of the metric Noloss Interaction by using equation 5 that would represent a non-risky interaction and hence no loss in financial terms to the trusting peer we get:

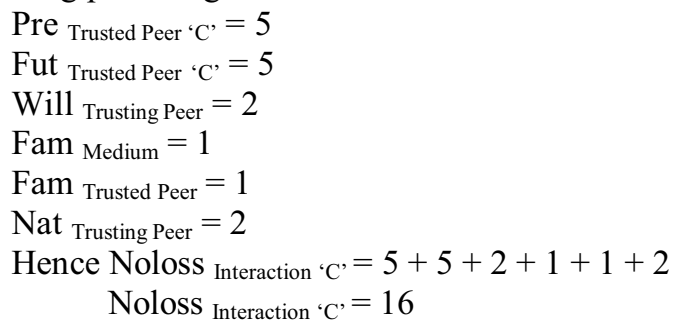

Utilizing equation 6 to determine in numerical terms the degree of possible loss that could be possible in the interaction we get:

Loss $_{\text {Interaction ' } \mathrm{C} \text { ' }}=\frac{16-13}{16}$

Loss ${ }_{\text {Interaction }}{ }^{\mathrm{C}} \mathrm{C}=0.1875$

Consequently determining the Financial Loss in an interaction according to equation 7

\section{Financial Loss in interacting with peer ' $C$ '$$
=(15000 * 0.1875)
$$$$
=\$ 2812.5
$$

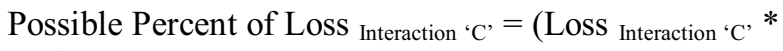
100)

Possible Percent of Loss Interaction $^{~} \mathrm{C}$ ' $=18.75 \%$

Hence the possible loss to the trusting peer ' $\mathrm{A}$ ' in interacting with the trusted peers ' $\mathrm{B}$ ' and ' $\mathrm{C}$ ' is $1875 \$$ and $2812.5 \$$ respectively. Based on the values achieved the trusting peer can decide with which peer to interact with.

\section{Conclusion}

In this paper we highlighted an important characteristic of Risk in any interaction, the impact in financial terms to the trusting peer's resources. The financial impact is usually the loss incurred to the trusting peer in interacting with the trusted peer. The trusting peer can firm its decision of interacting with the trusted peer, by analysing the loss to its resources in the interaction. In this paper we identified the factors by which the loss in the trusting peer's resources in the interaction can be determined. Further we defined the metrics by which those factors can be quantified and considered to determine the financial loss in an interaction. We then defined a methodology by which the trusting peer can determine the possible financial loss in interacting with the trusted peer and finally concluded the paper by explaining the methodology with an example.

\section{References}

[1] D.F. Cooper, 'The Australian and New Zealand Standard on Risk Management, AS/NZS 4360:2004', Tutorial Notes: Broadleaf Capital International Pty Ltd. Available: http://www.broadleaf.com.au/tutorials/Tut_Standard.pdf

[2] J.G. March and Z. Shapira, 'Managerial perspective on risk and risk taking',

Management Science, vol. 33, no. 11, pp. 1404-1418,1987 [3] N. Luhmann, 'Familiarity, confidence, trust: Problems and alt
Breaking Cooperative Relations, Basil Blackwell, New York, USA.

[4] R.C. Mayer, J.H. Davis and F.D. Schoorman, 'An interactive model for organizational trust', Academy of Management Review, vol. 20, no. 3, pp.709-734.

[5] D.M. Rousseau, S.B. Sitkin, R.S. Burt and C. Camerer, 'Not so different after all: A cross-discipline view of trust', Academy of Management Review, vol. 23, no. 3, pp. 391-404. [6] P. Sztompka, 'Trust: A sociological theory', Cambridge University Press, Cambridge, U.K.

[7] S. Grazioli and A. Wang, 'Looking without seeing: Understanding unsophisticated consumers success and failure to detect Internet deception', Proceedings of the International Conference on Information Systems, New Orleans, USA, December 16-19 200 pp 193-204.

[8] C. Cheung and M.K.O. Lee, 'Trust in Internet shopping: A proposed model and measurement instrument', Proceedings of the 2000 Americas Conference on Information Systems, Long Beach, CA, August 10-13 2000, , pp 681-689.

[9] O.K. Hussain, E. Chang, F.K.Hussain and T.S. Dillon, "A Methodology for Risk Measurements in e-Transactions", Special issue of International Journal of Computer System, Science and Engineering, CRL publishing Ltd, UK., To be published in March/April 2006.

[10] O.K Hussain, E.Chang, F.K. Hussain, T.S. Dillon and B. Soh, "Modeling the Risk [10] O.K Hussain, E.Chang, F.K. Hussain, T.S. Dillon and B. Soh, "Modeling the Risk
Relationships and Defining the Risk Set" Proceedings of CollECTeR Latam 2005, Chile, 35 October 2005, pp 1-9.

[11] O.K Hussain, E.Chang, F.K. Hussain, T.S. Dillon and B. Soh, "Context and Time Dependent Risk based Decision Making", Accepted for Publication in 20th IEEE International Conference on Advanced Information Networking and Applications (AINA 2006), Vienna, April 18-20 2006.

[12] O.K Hussain, E.Chang, F.K. Hussain, T.S. Dillon and B. Soh, "Predicting the Dynamic Nature of Risk", Accepted for Publication in the 4th ACS/IEEE International Conference on Computer Systems and Applications (AICCSA-06), Dubai/Sharjah, UAE, March 8-11 2006. [13] S. Greenland, 'Bounding analysis as an inadequately specified methodology', Risk Analysis, vol. 24, no. 5, pp. 1085-1092, 2004

[14] M. E. Orlowska, 'The Next Generation Messaging Technology - Makes Web Services Effectives', Proceedings of the Sixth Asia Pacific Web Conference, pp. 13-19, SpringerVerlag, Berlin Heidelberg 2004.

[15] C. Qu, and W. Nejdl, 'Interacting the Edutella/JXTA Peer-to-Peer Network with Web Services', Proceedings of the 2004 International Symposium on Applications and the Internet (SAINT'04), pp 67, 2004

[16] C. Schmidt, and M. Parashar, 'A Peer-to-Peer Approach to Web Service Discovery', World Wide Web Journal, Vol. 7, Issue 2, pp. 211-229, 2004.

[17] C. Schuler, R. Weber, H. Schuldt, and H. Schek, 'Scalable Peer-to-Peer Process Management - The OSIRIS Approach', Proceedings of the IEEE International Conference on Web Services, San Diego, USA, pp.26, 2004.

[18] M. Ripeanu, 'Peer-to-Peer Architecture Case Study: Gnutella Network', Proceedings of the First International Conference on Peer-to-Peer Computing , pp 99-100, 2001.

[19] M.P. Papazoglou, B.J. Kramer, and J. Yang, 'Leveraging Web-Services and Peer-toPeer Networks', Springer-Verlag Berlin Heidelberg 2003.

[20] O.K Hussain, E.Chang, F.K. Hussain, T.S. Dillon and B. Soh, "Context Based Riskiness Assessment", Proceedings of IEEE TENCON 2005, pp. 352-356, Melbourne,
22-24 